\title{
Point Spread Function of EUSO-TA detector
}

\author{
Z. Plebaniak ${ }^{* a \dagger}$, J. Szabelski ${ }^{a}$, T. Wibig ${ }^{a, b}$, L.W. Piotrowski ${ }^{c}$ for the JEM-EUSO \\ Collaboration \\ ${ }^{a}$ National Centre for Nuclear Research, Astrophysics Division, Cosmic Ray Laboratory, \\ ul. 28 Pułku Strzelców Kaniowskich 69, 90-558 Łódź, Poland, E-mail: zp@ zpk • u. lodz •pl. \\ ${ }^{b}$ Faculty of Physics and Applied Informatics, University of Łódz, \\ ul. Pomorska 149/153, 90-236 Łódź, Poland. \\ ${ }^{c}$ RIKEN, Wako, Japan
}

EUSO detectors are high-resolution fluorescence telescopes dedicated for measurements of ultrahigh energy cosmic rays (UHECR). EUSO-TA is a experiment dedicated for testing EUSO detectors and measurements of UHECR in coincidence with Telescope Array (TA) experiment. EUSOTA is located in front of the Black Rock Mesa (BRM) detectors of the TA experiment. During a few campaigns in 2015 and 2016 signals coming from stars, airplanes, meteors, laser shots and cosmic rays were registered. The main goal of this work is determination of Point Spread Function of EUSO detectors which plays very important role in the interpretation of registered signal.

35th International Cosmic Ray Conference - ICRC2017

10-20 July, 2017

Bexco, Busan, Korea

* Speaker.

${ }^{\dagger}$ We dedicate this proceeding to Yoshiya Kawasaki and Jacek Karczmarczyk, who passed away in 2016. 


\section{Introduction}

EUSO-TA is a ground based fluorescence detector, located at Black Rock Mesa site (Fig. 1, left), where one of the three fluorescence detectors of Telescope Array (TA) experiment. The idea of this experiment is to test and calibrate EUSO detectors by measurements in coincidence with TA.
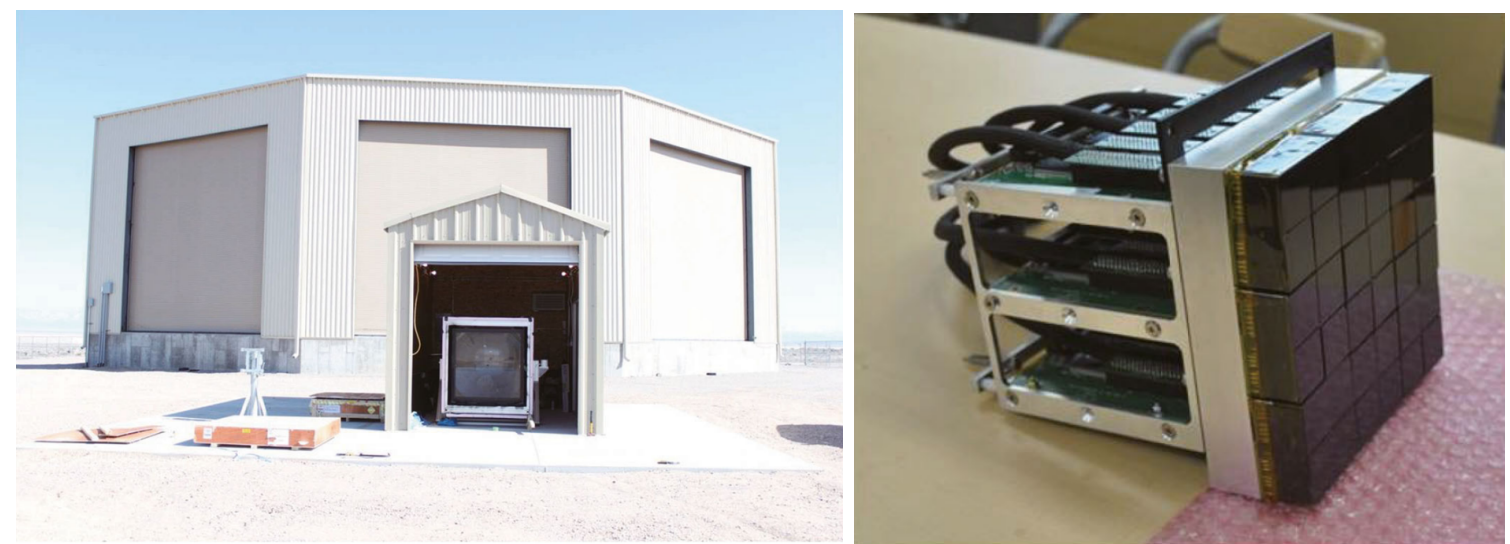

Figure 1: EUSO-TA building in front of Telescope Array Fluorescence Detector station at Black Rock Mesa site (left) and PDM frame with PMT's array used in experiment (right) [1]

The EUSO-TA optics consists of two $1 \mathrm{~m}^{2}$ flat Fresnel PMMA lenses (Fig. 2) [2]. This system is focusing light in a $10.6^{\circ}$ field of view on one Photo Detector Module (PDM) shown in Fig. 1, right. More details of EUSO-TA optical system are described in [2, 3]. Focal surface of PDM is a matrix of 36 Multi Anode (64 channels) Photomultiplier Tubes (MAPMT) which gives 2304 pixels. Measurement is realized by counting registered photoelectrons. Gate Time Unit (GTU) of equal $2.5 \mu \mathrm{s}$ gives possibility for registration of fast moving UV signals induced by an Extensive Air Shower passing through the Earth's atmosphere.

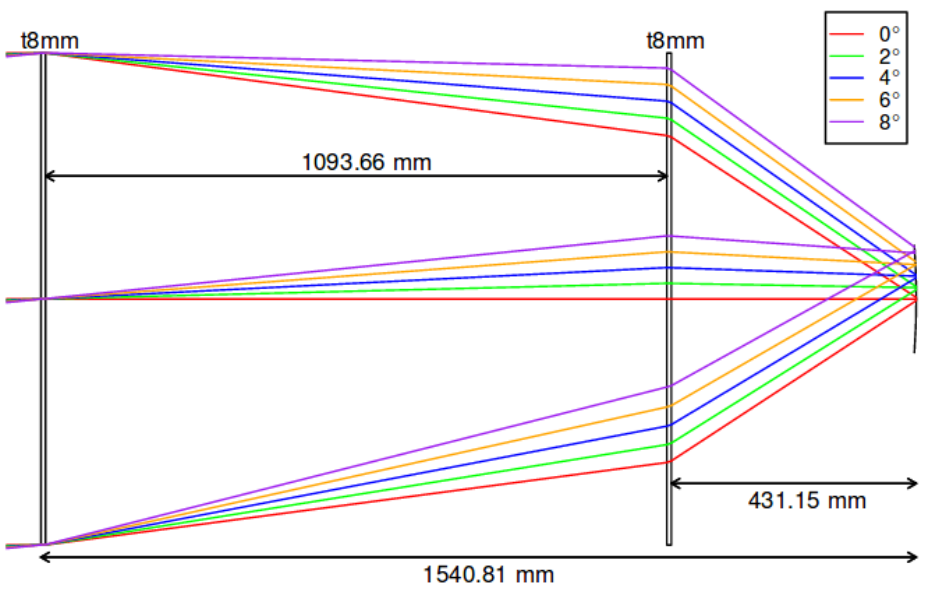

Figure 2: The optics design of EUSO-TA. 
During operations of EUSO-TA, signals emitted by Central Laser Facility of TA can be registered and used for calibration and estimation of weather condition. EUSO-TA experiment, which is sensitive to UV light in wavelength range between $290 \mathrm{~nm}$ and $400 \mathrm{~nm}$ can observe also signals from planes and stars. Used optical system should allow for registration of signals coming from stars in $3 \times 3$ pixels spot.

The goals of this work is determination of Point Spread Function (PSF) describing registered star signals by 2-dimensional Gaussian function. Stars registered during EUSO-TA campaign in October 2016 were analyzed. Registered stars have been identified using Hipparcos catalogue [4]. Examples of a few registered stars are shown in Fig. 3. Pictures show image of the sky evolution in time. Difference between left and right pictures is 700 packets. Each picture represents data for one packet which is related to average signal of 128 GTU's.

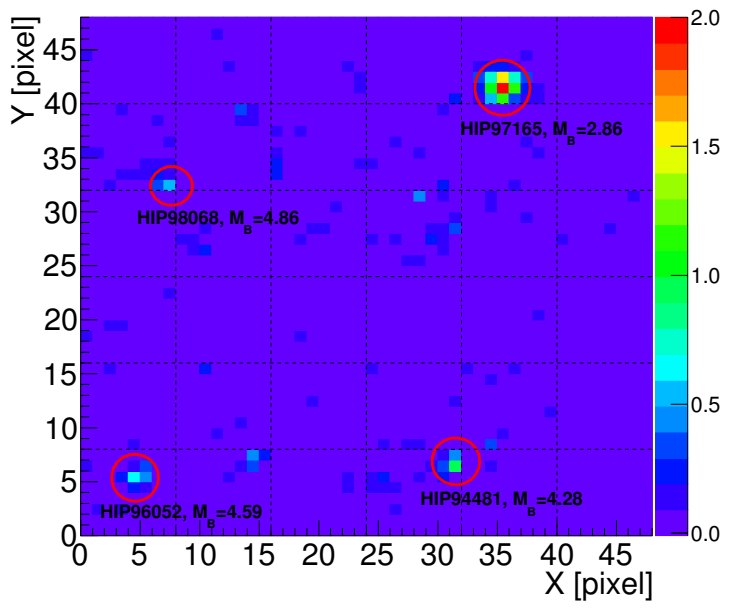

(a) Packet No. 400, 2016-10-05 08:35:16 UTC

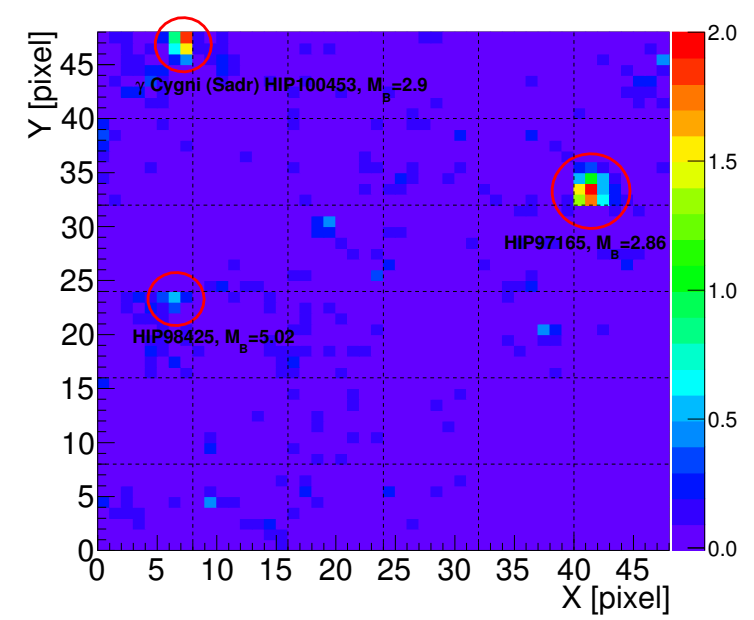

(c) Packet No. 1800, 2016-10-05 08:48:27 UTC

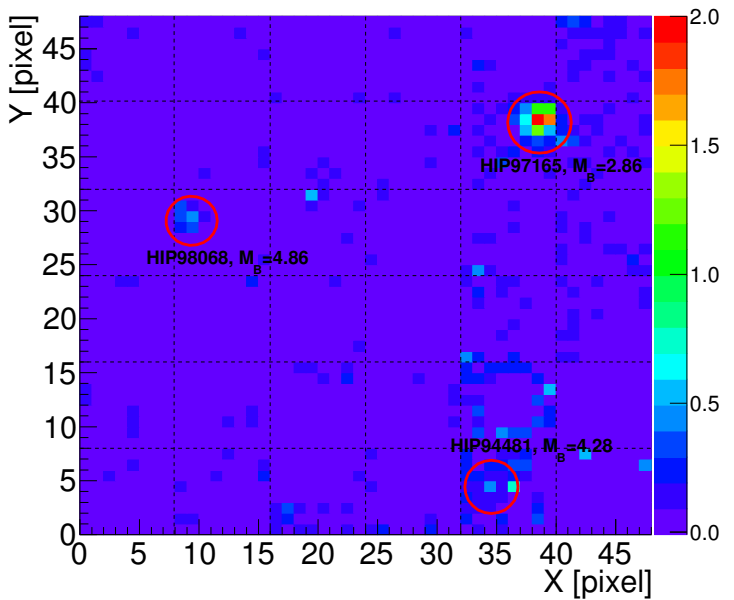

(b) Packet No. 1100, 2016-10-05 08:41:12 UTC

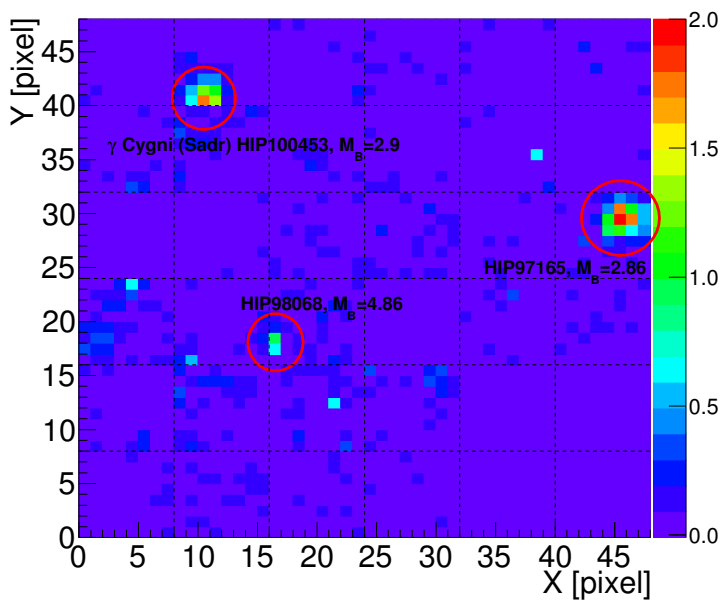

(d) Packet No. 2500, 2016-10-05 08:56:14 UTC

Figure 3: Image of the sky with stars registered during EUSO-TA campaign in October 2016. Pictures show results for four packets registered October 5th. Distance beetween following pictures is 700 packets what is related to about 7 minutes (depends on triggering). 
Accuracy of star detection and identification strongly depends on weather conditions and UV background. Signals taken into acount were registered during non-cloudy or partially cloudy conditions when star was not covered by cloud. Analysis confirmed that EUSO-TA can recognize stars with magnitude in Hipparcos B filter $M_{B}<6.0$ [5] but only objects with $M_{B}<4.5$ are bright enough to be successfully fitted using the discussed procedure.

\section{Fitting Method}

EUSO-TA mesurements are externally triggered by signal from TA experimment. After TA trigger 128 frames (GTU's) received from EUSO-TA are stored as one packet. TA trigger rate is more than $2 \mathrm{~Hz}$. The time distance between the first and the last frame in packet is 0.32 milliseconds which means that star position does not move on focal surface in a significant way in packet range. For present analysis all frames in packet were summed up, then background was corrected and pixels equalized. After this operation visible signals from stars were fitted using 2D Gaussian function 2.1.

$$
f(x, y)=\mathbf{A} \exp \left(-\left(\frac{X^{\prime 2}}{2 \sigma_{\mathbf{x}}^{2}}+\frac{Y^{\prime 2}}{2 \sigma_{\mathbf{y}}^{2}}\right)\right)
$$

where $X^{\prime}$ and $Y^{\prime}$ are defined using rotation matrix as follows:

$$
\left[\begin{array}{l}
X^{\prime} \\
Y^{\prime}
\end{array}\right]=\left[\begin{array}{cc}
\cos \phi & -\sin \phi \\
\sin \phi & \cos \phi
\end{array}\right] \times\left[\begin{array}{l}
x-\mathbf{x}_{\mathbf{0}} \\
y-\mathbf{y}_{\mathbf{0}}
\end{array}\right]
$$

Parameters $x_{0}$ and $y_{0}$ represent positions of described signal. For different $\sigma_{x}$ and $\sigma_{y}$ parameters, projection of $f(x, y)$ on XY plane is an ellipse. Fit function can be rotated around $x_{0}$ and $y_{0}$ values on XY plane defining rotation angle $\phi$ - fifth parameter of function as an angle between major axis of an ellipse and OY axis.

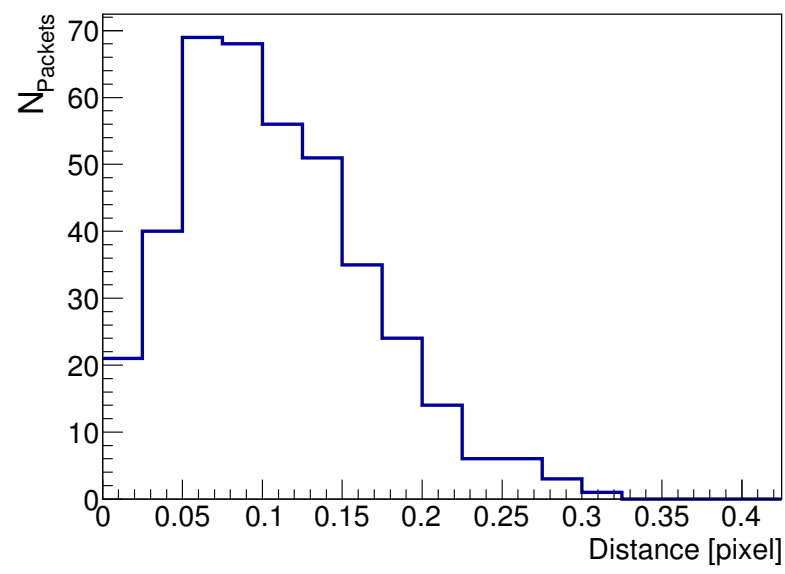

(a) Distance between real and calculated star position.

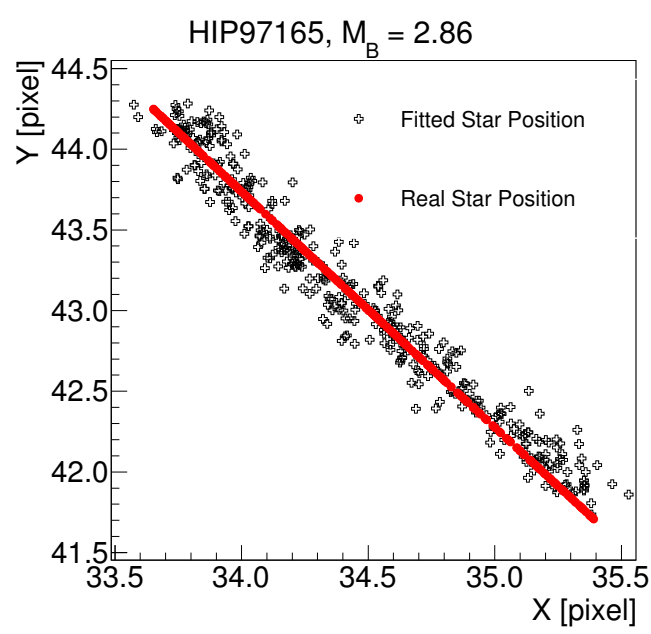

(b) Comparison of calculated track of star with positions found by algorithm.

Figure 4: Accuracy of star position determination using fitting algorithm. 
Accuracy of used fitting algorithm is shown in Fig. 4 on the example of determining the trace of a star HIP97165 with $M_{B}=2.86^{m}$. Comparison between real star positions (red markers), calculated using ephemeris and Hipparcos catalogue, and positions obtained by fitting (black markers) is presented in Fig. 4, right. Histogram presented in Fig. 4, left shows distribution of differences between real and obtained star position. Both figures concern analysis of 400 packets. During 5 nights of mesurements in October 2016 around 25000 packets with signals clear enough for analysis have been registered. Fig. 5 shows crossing of EUSO-TA focal surface by signals from stars with different brightness. Most of useful signals come from stars with $3.0<M_{B}<4.0$. All algorithms for this analysis have been prepared using ROOT framework [6], fitting of stars signals have been performed using MINUIT with Minimum Likelihood Estimation.

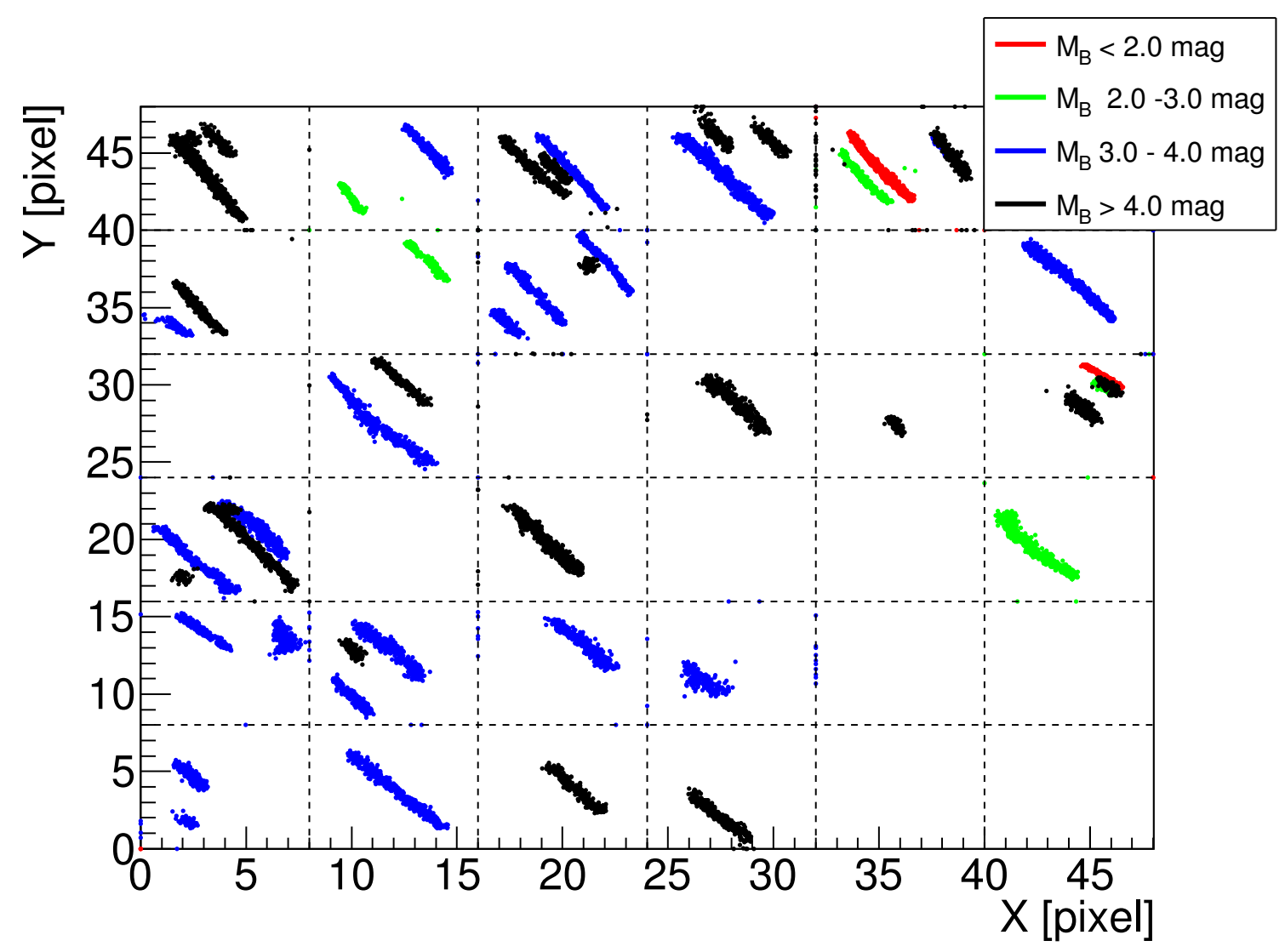

Figure 5: Positions of all stars detected during EUSO-TA mesurements in October 2016

\section{Results}

PSF of EUSO-TA detector is described in general by two $\sigma$ parameters appearing in Eq. 2.1. As fitting function can be rotated, we distinguish $\sigma_{\text {Minor }}$ and $\sigma_{\text {Major }}$ parameters describing widths of minor and major ellipse axis instead of $\sigma_{x}$ and $\sigma_{y}$ parameters. In Fig. 6 distributions of $\sigma_{\text {Minor }}$ and $\sigma_{\text {Major }}$ for each PMT on focal surface separetely are presented. Red color represents $\sigma_{\text {Major }}$ distributions while black is related to $\sigma_{\text {Minor }}$. We can see that there are no outstanding PMT's al- 
though statistics is relatively low. Empty places represent PMT's which were not working properly during observations or in which clear signals from stars have been not registered during analyzed measurements.
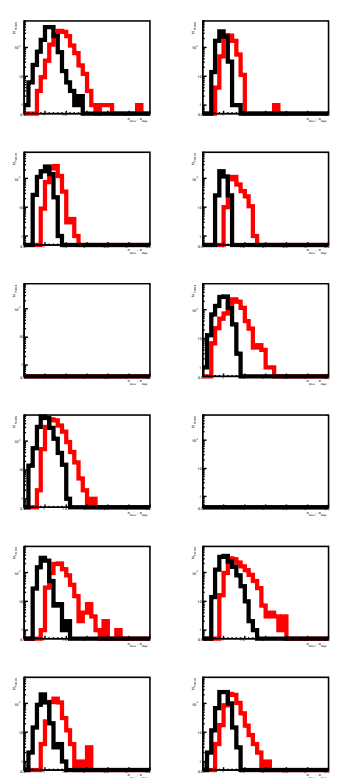
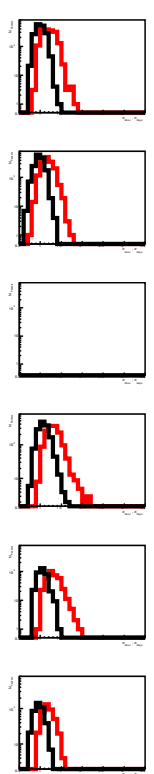
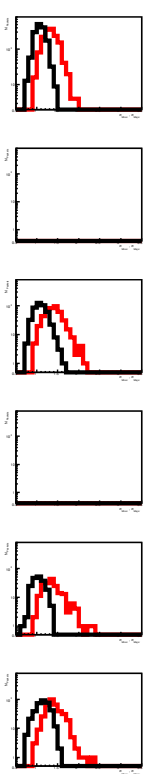
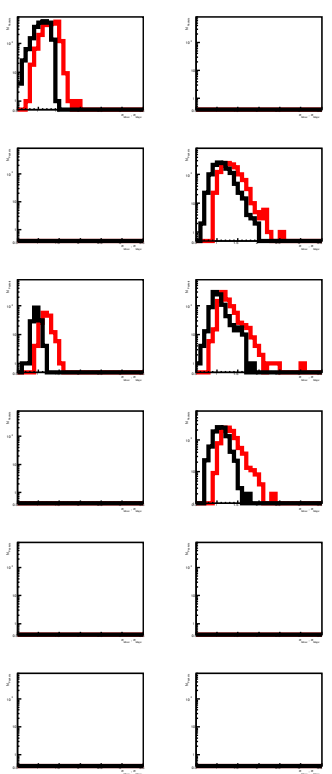

Figure 6: $\sigma$ parameter distributions for each PMT. On the X axis: $\sigma_{\text {Minor }}-$ black histogram lines, and $\sigma_{\text {Major }}$ - red histogram lines in pixels in the range between 0.5 and 3.5. On the $\mathrm{Y}$ axis is the number of packets with maximum at 800 .

Finally we present global $\sigma$ distributions resulting from the summation of fitting results in all PMT's (Fig. 7). This way we obtained average values of $\sigma_{\text {Minor }}$ and $\sigma_{\text {Major }}$ parameters and corresponding to them FWHM parameters. Results are presented in Tab. 1.

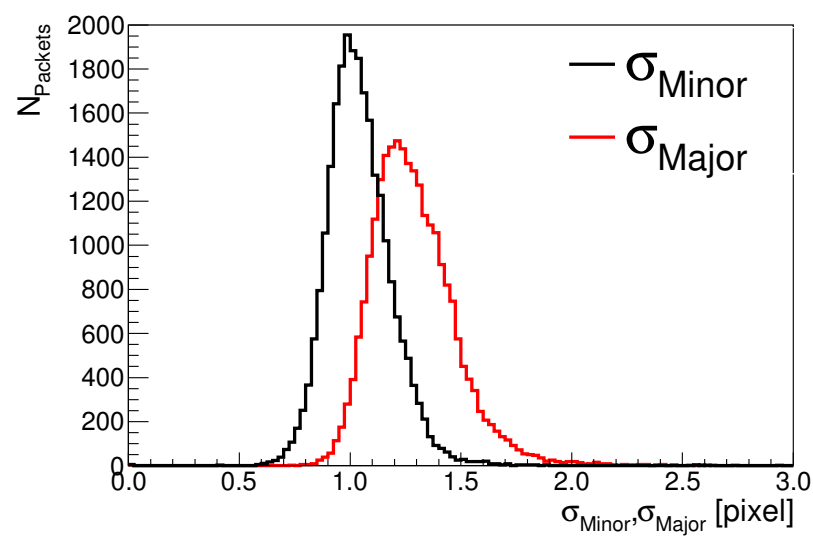

Figure 7: Total $\sigma$ distribution including all stars fitted during analysis 
Table 1: Average $\sigma$ parameters of major and minor ellipse axis and related FWHM values

\begin{tabular}{|l|c|c|}
\hline & $\sigma[$ pixel $]$ & FWHM [pixel] \\
\hline$\sigma_{\text {Major }}$ & $1.27 \pm 0.03$ & $2.98 \pm 0.07$ \\
\hline$\sigma_{\text {Minor }}$ & $1.04 \pm 0.02$ & $2.46 \pm 0.04$ \\
\hline
\end{tabular}

Additionaly we present distribution of percentage of the signal from a star in all PSF pixels (Fig. 8). Figure was created by summing histograms of counts number during fitting procedure. Central pixel was placed always in fitted centre of the signal, summing was performed in the range \pm 3 pixels. Histogram and numbers represent percentage of the signal, while the sum of all displayed pixels is normalized to $100 \%$. Figure presents results for summing of 100 packets for one selected star.

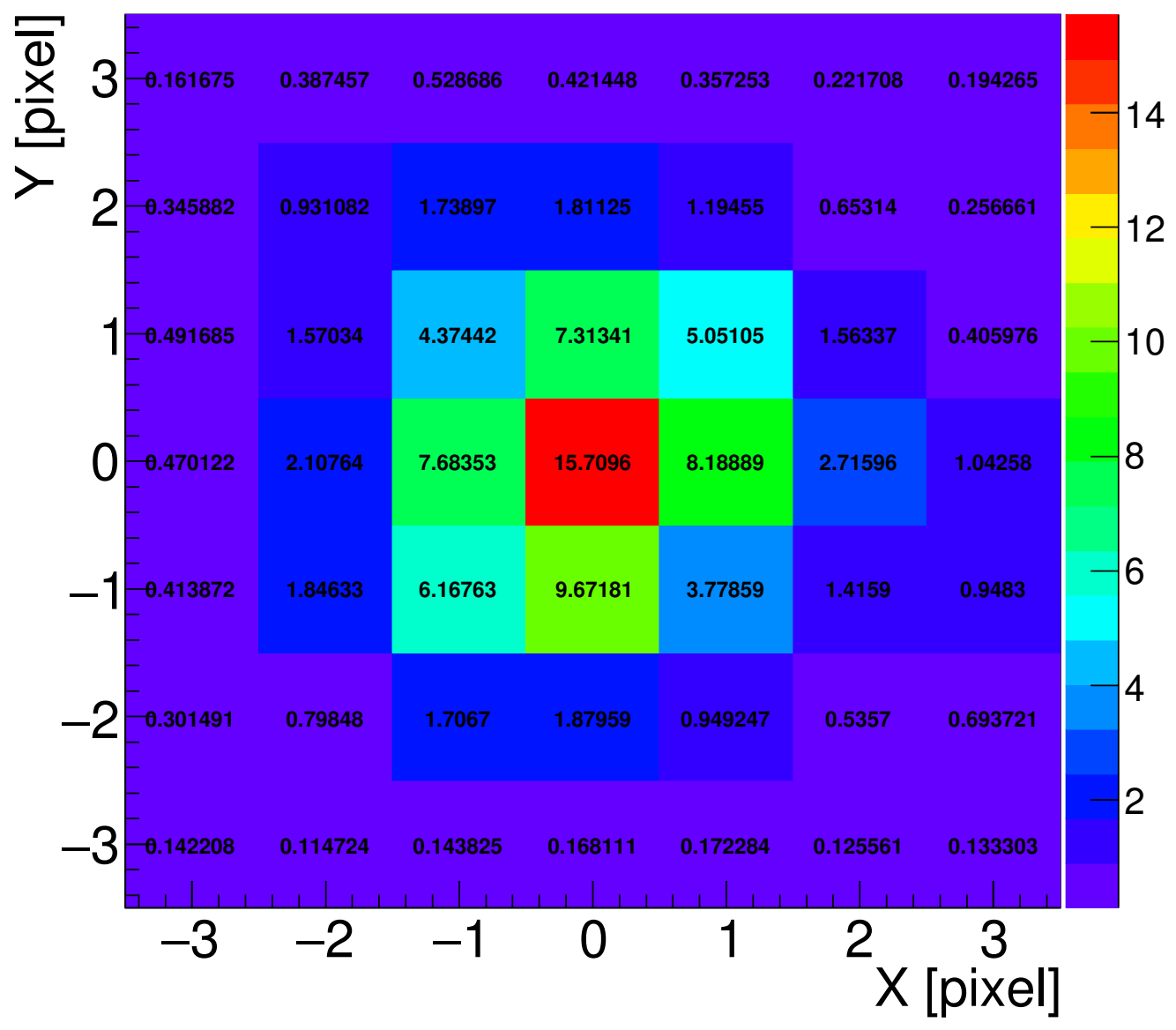

Figure 8: Percentage of signal in central and nearby pixels for a sample star. 


\section{Conclusions}

During this work almost 25000 clear signals from stars, registered during EUSO-TA measurements in October 2016 have been analyzed. Signals from stars have been fitted by 2D Gaussian function to find the form of registered picture and finally to estimate parameters of PSF for EUSOTA detector. As a result we report that signals from stars are elliptical with $\sigma_{\text {Minor }}=1.04$ pixels and $\sigma_{\text {Major }}=1.27$ pixels which gives FWHM parameters 2.46 and 2.98 respectively. This is in agreement with laboratory tests of used optical system [2] and measurements made for similar device used during the EUSO-Balloon first flight [7]. Analysis shows that signal registered from point source like a star is visible in a spot with sizes less than $3 \times 2.5$ pixels with approximately $15 \%-$ $22 \%$ of signal in central pixel. This meets requirements for registration of UHECR showers which is the main goal of EUSO detectors. Assymetry of gaussian which is constant all over the PDM is most likely due to a misalignment of the PDM and lenses planes.

Acknowledgments: This work was supported by grant no 2015/19/N/ST9/03708 funded by National Science Centre in Poland.

\section{References}

[1] Y. Kawasaki, L. W. Piotrowski et al. for JEM-EUSO Collaboration, Ground-based tests of JEM-EUSO components at the Telescope Array site, "EUSO-TA", Exp. Astron., 40, 301-314, doi:10.1007/s10686-015-9441-6, 2015

[2] Y. Takizawa et al. for JEM-EUSO Collaboration, Proc. 33rd ICRC, The TA-EUSO and EUSO-Balloon optics design (2013)

[3] Y. Hachisu et al. for JEM-EUSO Collaboration, Proc. 33rd ICRC, Manufacturing of the TA-EUSO and the EUSO-Balloon lenses (2013)

[4] M. A. C. Perryman et al., The HIPPARCOS Catalogue, Astronomy and Astrophysics, 323 (1997), L49-L52

[5] M. Casolino et al. for JEM-EUSO Collaboration, Proc. 34th ICRC, EUSO-TA, a ground telescope at the Telescope Array to test JEM-EUSO detector performance (2015)

[6] http://www.root.cern.ch

[7] C. Catalano et al. for JEM-EUSO Collaboration, Proc. 34th ICRC, Performance of EUSO-Balloon optics. 